\title{
A Mathematical Model and Error Analysis of Shearer Cutting Path Based on Its Attitude
}

\author{
Shi-bo Wang $\mathbb{D D}^{1,2}$ Shijia Wang, ${ }^{1,2}$ and Zhaoliang Ge \\ ${ }^{1}$ School of Mechanical \& Electrical Engineering, China University of Mining \& Technology, Xuzhou 221116, China \\ ${ }^{2}$ Intelligent Mining Equipment Collaborative Innovation Center, China University of Mining \& Technology, Xuzhou 221116, China
}

Correspondence should be addressed to Shi-bo Wang; wangshb@cumt.edu.cn

Received 5 June 2018; Accepted 30 August 2018; Published 17 September 2018

Academic Editor: Francesc Pozo

Copyright (C) 2018 Shi-bo Wang et al. This is an open access article distributed under the Creative Commons Attribution License, which permits unrestricted use, distribution, and reproduction in any medium, provided the original work is properly cited.

The horizon control system is the key technology in the automation of a shearer. The achievement of accurate shearer cutting path plays an important role for horizon control. A mathematical model of cutting path in the local geographic coordinate frame was built. Error analysis based on genetic algorithm (GA) was studied to guarantee the accuracy of the shearer cutting path. Parameters from a MG1000/2660-WD shearer and data from a working face were used to obtain the shearer cutting path with reference to the local geographic coordinate frame. Also, with error analysis based on GA, the desired sensors were chosen, which allowed coordinate position errors of a shearer's cutting path to be less than $0.01 \mathrm{~m}$. The desired accuracies of the inertial navigation system and encoders mounted on the different shearers used in thin seam, medium-thickness seam, and thick seam were calculated.

\section{Introduction}

Longwall automation can deliver benefits to the industry in terms of increased productivity and improvement in conditions for current on-face workers, particularly by removing them from hazards. Horizon control of a shearer is a vital part of longwall mining automation $[4,5]$. The goal of horizon control is to automatically maintain the longwall shearer's cutting trace within the coal seam so that the mining resource can be optimally extracted [6]. The cutting trace is mainly determined by the height of the drum and the attitude of a shearer. In the practical mining workface, the height of drum is manually adjusted according to manual observations in order to avoid interference between the drum and the roof and floor of the workface. A geometric track cuttingmemory method has been applied in several mining shearers, but this approach only achieves the desired in-seam control over a small number of shearer cycles as it cannot take into account the higher than first-order trends and shortterm variations in the seam horizon [7]. Some researchers have proposed drum height adjustment based on a coal interface detection method using natural gamma radiation, vibration signal, thermal infrared rays, and other pieces of sensor information to identify coal and rock as mentioned in
[6-10]. However, the coal interface detection methods have never been successfully applied because of the complicated and unpredictable geological conditions in a coal-mining face.

Previous automation attempts have in large part been stymied by the inability to accurately determine the threedimensional path of the longwall shearer as it systematically progresses through the coal panel. Without this information there is no absolute reference for controlling the motion of the equipment, and reliable, sustained automation cannot be achieved [11]. The Australian Coal Association Research Program (ACARP) commissioned a three-year 'Landmark' project. In the project, inertial navigation technology has, for the first time, allowed the position of a longwall shearer to be mapped in three dimensions.

It is important to obtain the cutting path of a shearer for maintaining the longwall shearer's cutting trace within the coal seam. In this paper, a mathematical model based on the attitude of a shearer was built, with which the position of the drums of a shearer relative to the local geographic frame was determined. Considering that the performance of the automated horizon control system is critically dependent on the accuracy of a shearer's cutting path, the error of this model, induced by the errors of attitude sensors, is 


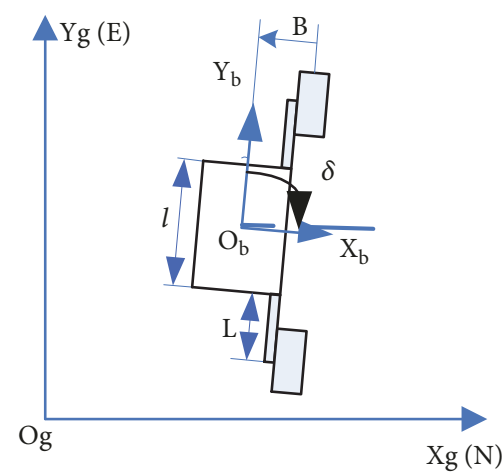

(a)

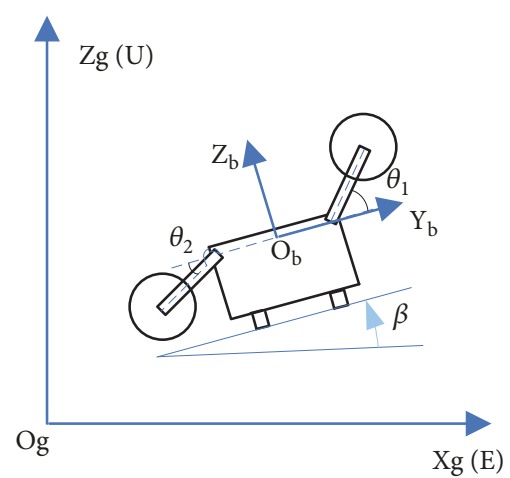

(b)

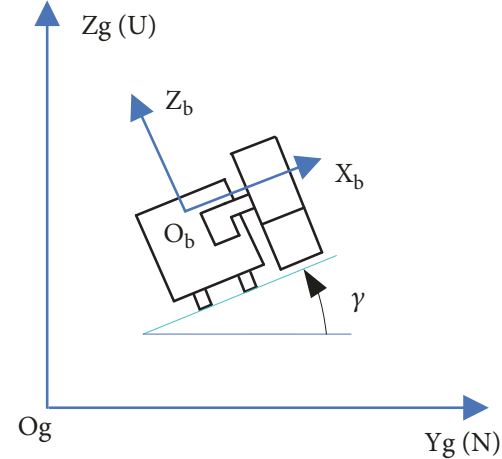

(c)

FIgURE 1: A depiction of a shearer's heading, pitch and roll angles, and the relationship between the shearer frame $\left(\mathrm{X}_{b}, \mathrm{Y}_{b}\right.$, and $\left.\mathrm{Z}_{b}\right)$ and local geographic frame $\left(\mathrm{X}_{g}, \mathrm{Y}_{g}\right.$, and $\left.\mathrm{Z}_{g}\right)$.

analyzed with genetic algorithm (GA). The error analysis of the model provides the theoretical basis for choosing suitable sensors.

\section{Model of the Absolute Position of the Shearer Drums}

The variations of coordinates of a shearer, including heading angle $\delta$, pitch angle $\beta$, rolling angle $\gamma$, and the angle between the arms and the mainframe $\theta_{1}, \theta_{2}$, are the most important factors influencing the cutting path, as shown in Figure 1. An inertial navigation system (INS) mounted onto the mainframe of a shearer not only measured the attitude and heading of a shearer (including heading angle $\delta$, pitch angle $\beta$, and rolling angle $\gamma$ ) but also measured the three-dimensional position with the aid of an odometer [12]. The angle between the arms and mainframe was measured by axial encoders. The INS and axial encoders fixed to the shearer are shown in Figure 2.

According to the principle of inertial navigation, the shearer frame is referred to as a body frame, as shown in Figure 2. The origin of the shearer frame coincides with the gravity centre of the shearer. The $\mathrm{X}_{b}$-axis, $\mathrm{Y}_{b}$-axis, and $\mathrm{Z}_{b}$-axis are aligned with the pitch, roll, and yaw axes, respectively, of the shearer in which the navigation system is installed. A local geographic frame was built which has its origin at the location of the start point of the longwall mining face. The $\mathrm{X}_{g}$-axis, $\mathrm{Y}_{g^{-}}$ axis, and $Z_{g}$-axis are aligned in the directions of east, north, and the local vertical (up). As illustrated in Figures 1 and 3, the along-face direction is $\mathrm{X}_{g}(\mathrm{E})$, the face-advance (retreat) direction is $\mathrm{Y}_{g}(\mathrm{~N})$, and the vertical direction is $\mathrm{Z}_{g}(\mathrm{U})$ [13].
As the reference point of the position of a shearer mainframe, $\mathrm{O}_{b}$, is represented by, with respect to the local geographic frame $g$,

$$
\mathbf{P}_{O}^{g}=\left[\begin{array}{lll}
X_{O} & Y_{O} & Z_{O}
\end{array}\right]^{T}
$$

$\mathbf{P}_{1}^{b}$ and $\mathbf{P}_{2}^{b}$ are taken as the centre position of the drums of a shearer with respect to the shearer frame $b$. They are described as follows:

$$
\begin{aligned}
& \mathbf{P}_{\mathbf{1}}^{\mathbf{b}}=\left[\begin{array}{c}
B \\
L \cos \theta_{1}+\frac{l}{2} \\
L \sin \theta_{1}
\end{array}\right], \\
& \mathbf{P}_{\mathbf{2}}^{\mathbf{b}}=\left[\begin{array}{c}
B \\
-L \cos \theta_{2}-\frac{l}{2} \\
L \sin \theta_{2}
\end{array}\right]
\end{aligned}
$$

where $B$ is the centre distance between shearer drum and shearer mainframe, $l$ is the length of the mainframe of a shearer, and $L$ is the length of the arm, as presented in Figure 1 . The subscripts 1 and 2 are the left and right drums, respectively.

According to the principle of transformation between body frame and local geographic frame mentioned in [14], the relationship between the shearer frame $b$ and the local geographic frame $g$ is given as follows:

$$
\mathbf{P}_{i}^{g}=\mathbf{P}_{\mathbf{0}}^{g}+\mathbf{R}_{\mathbf{b}}^{g} \cdot \mathbf{P}_{i}^{b} \quad(i=1,2)
$$

where the transformation matrix from the shearer frame $b$ to the local geographic frame $g$ is

$$
\mathbf{R}_{\mathbf{b}}^{\mathbf{g}}=\left[\mathbf{R}_{\mathbf{g}}^{\mathbf{b}}\right]^{-1}=\left[\begin{array}{ccc}
\cos \delta & \sin \delta & 0 \\
-\sin \delta & \cos \delta & 0 \\
0 & 0 & 1
\end{array}\right] \bullet\left[\begin{array}{ccc}
\cos \gamma & 0 & \sin \gamma \\
0 & 1 & 0 \\
-\sin \gamma & 0 & \cos \gamma
\end{array}\right] \bullet\left[\begin{array}{ccc}
1 & 0 & 0 \\
0 & \cos \beta & -\sin \beta \\
0 & \sin \beta & \cos \beta
\end{array}\right]
$$




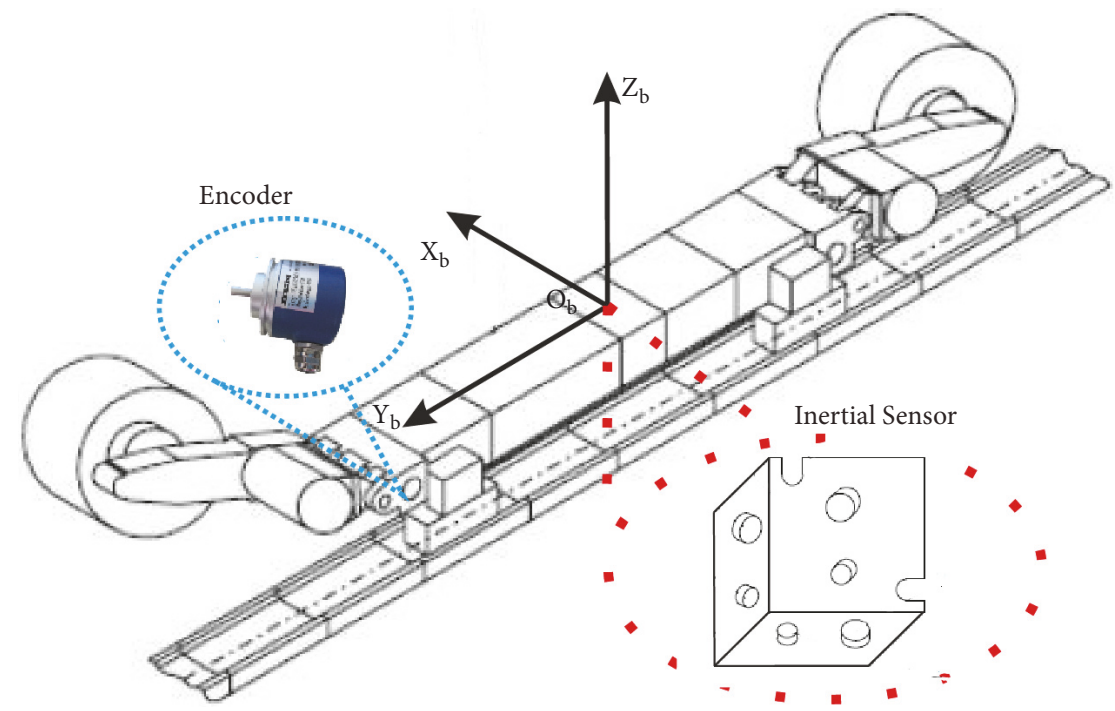

FIgURE 2: The shearer frame fixed with the INS and axial encoders.

Combining (1)-(4), we get the coordinates of the positions of the shearer drums with respect to the local geographic frame $g$, as follows:

$$
P_{1}^{g}=\left\{\begin{array}{c}
X_{O}+B \cos \delta \cos \gamma+\left(\frac{l}{2}+L \cos \theta_{1}\right)(\sin \delta \cos \beta+\cos \delta \sin \gamma \sin \beta) \\
+L \sin \theta_{1}(-\sin \delta \sin \beta+\cos \delta \sin \gamma \cos \beta) \\
Y_{O}-B \sin \delta \cos \gamma+\left(\frac{l}{2}+L \cos \theta_{1}\right)(\cos \delta \cos \beta-\sin \delta \sin \gamma \sin \beta) \\
-L \sin \theta_{1}(\cos \delta \sin \beta+\sin \delta \sin \gamma \cos \beta) \\
Z_{O}-B \sin \gamma+\left(\frac{l}{2}+L \cos \theta_{1}\right) \sin \beta \cos \gamma+L \sin \theta_{1} \cos \beta \cos \gamma
\end{array}\right\}
$$

\section{Error Analysis}

It is known from (5) and (6) that the position error of the shearer drums was mainly affected by the angle error of $\delta, \beta, \gamma, \theta_{1}$, and $\theta_{2}$ which was determined by the sensors including the INS and axial encoders. Usually, the price of these sensors increases with the improvement in their accuracy. Hence, INS and axial encoders with reasonable accuracy were chosen according to the tolerance limits of three-dimensional coordinates with absolute error. 


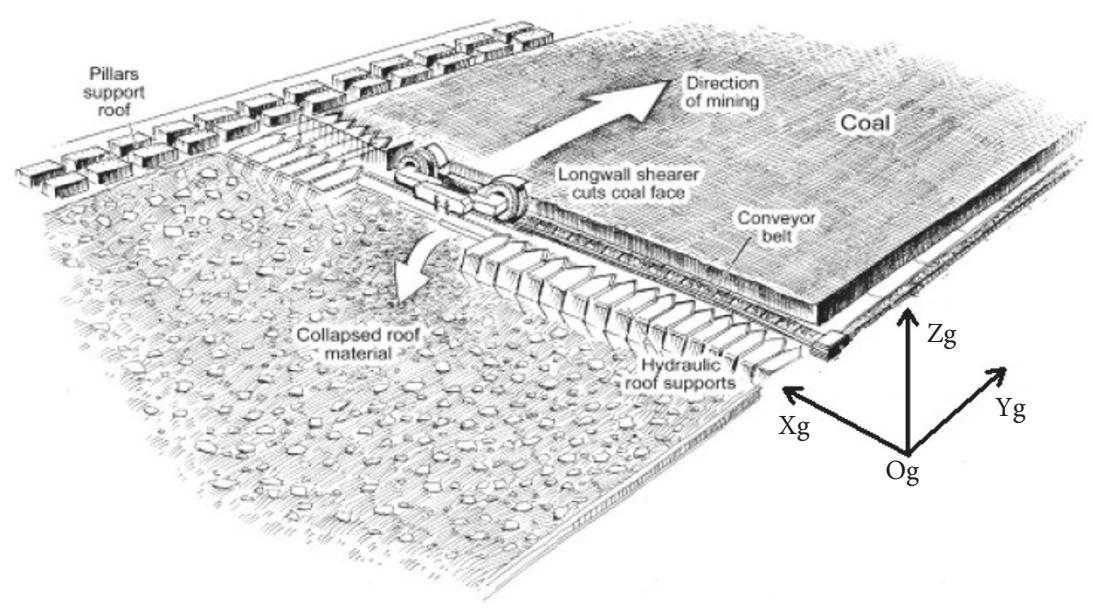

FIgURE 3: Typical longwall panel configuration and the local geographic frame [1].

GA is an artificial search method motivated by natural principles and the concept of survival of the fittest. Each independent variable in the GA is represented by a gene sequence and each solution is described as a chromosome. Compared with the general optimization algorithm, GA is a global search algorithm, which searches for multiple points with probability mechanism. So that a better global optimization value can be obtained. The mathematical model established in this paper is a kind of multiparameter constraining optimization and nonmonotonic problem. So GA algorithm was selected for analyzing the relation between the error in the cutting path and the accuracy of the sensors. The workflow of the error analysis is shown in Figure 4.

3.1. Objective Functions and Constraint Conditions. The error of absolute positions of shearer drums can be analyzed by derivation with an error transferring formula $[15,16]$ as follows:

$$
\Delta \mathbf{P}_{i}^{\mathbf{g}}=\left[\left|\frac{\partial \mathbf{P}_{i}^{\mathbf{g}}}{\partial \delta}\right|\left|\frac{\partial \mathbf{P}_{i}^{\mathbf{g}}}{\partial \beta}\right|\left|\frac{\partial \mathbf{P}_{i}^{\mathbf{g}}}{\partial \gamma}\right|\left|\frac{\partial \mathbf{P}_{i}^{\mathbf{g}}}{\partial \theta_{i}}\right|\right]\left[\begin{array}{c}
\Delta \delta \\
\Delta \beta \\
\Delta \gamma \\
\Delta \theta_{i}
\end{array}\right]_{(i=1,2)}
$$

where $\Delta \mathbf{P}_{\mathrm{i}}^{\mathrm{g}}, \Delta \delta, \Delta \beta, \Delta \gamma$, and $\Delta \theta_{i}$ are errors in $\mathbf{P}_{\mathrm{i}}^{\mathrm{g}}, \delta, \beta, \gamma$, and $\theta_{i}$, respectively. The angles $\beta$ and $\gamma$ are measured by the INS, so we can assume that the errors of $\beta$ and $\gamma$ are equal; namely, $\Delta \beta=\Delta \gamma$. The angles of $\theta_{1}$ and $\theta_{2}$ are measured by two axial encoders, so the errors in $\theta_{1}$ and $\theta_{2}$ are equal; namely, $\Delta \theta_{1}=\Delta \theta_{2}=\Delta \theta$. So (7) can be expressed in the form of

$$
\Delta \mathbf{P}_{i}^{\mathbf{g}}=\left[\left|\frac{\partial \mathbf{P}_{i}^{\mathbf{g}}}{\partial \delta}\right|\left|\frac{\partial \mathbf{P}_{i}^{\mathbf{g}}}{\partial \beta}\right|+\left|\frac{\partial \mathbf{P}_{i}^{\mathbf{g}}}{\partial \gamma}\right|\left|\frac{\partial \mathbf{P}_{i}^{\mathrm{g}}}{\partial \theta_{i}}\right|\right]\left[\begin{array}{c}
\Delta \delta \\
\Delta \beta \\
\Delta \theta_{i}
\end{array}\right]
$$

where

$$
\begin{aligned}
& K_{i}^{\max }=\left[\left|\frac{\partial \mathbf{P}_{i}^{\mathbf{g}}}{\partial \delta}\right|\left|\frac{\partial \mathbf{P}_{i}^{\mathbf{g}}}{\partial \beta}\right|+\left|\frac{\partial \mathbf{P}_{i}^{\mathbf{g}}}{\partial \gamma}\right|\left|\frac{\partial \mathbf{P}_{i}^{\mathbf{g}}}{\partial \theta_{i}}\right|\right]
\end{aligned}
$$

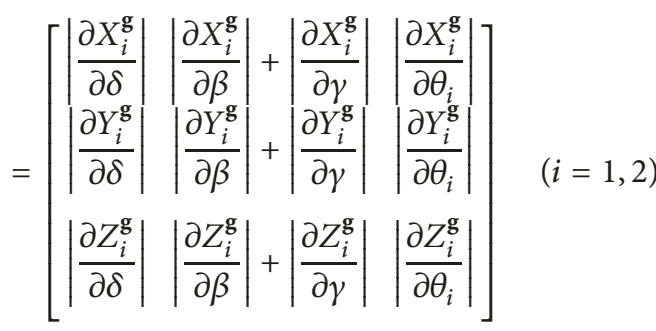

where $K_{i}^{\max }$ is the error coefficient matrix, whose elements will be treated as objective functions. Maximum values of the objective functions will be yielded with GA.

$$
\begin{array}{r}
{\left[\begin{array}{c}
\Delta \delta \\
\Delta \beta \\
\Delta \theta_{i}
\end{array}\right]=\left[\left|\frac{\partial \mathbf{P}_{i}^{\mathbf{g}}}{\partial \delta}\right|\left|\frac{\partial \mathbf{P}_{i}^{\mathbf{g}}}{\partial \beta}\right|+\left|\frac{\partial \mathbf{P}_{i}^{\mathbf{g}}}{\partial \gamma}\right|\left|\frac{\partial \mathbf{P}_{i}^{\mathbf{g}}}{\partial \theta_{i}}\right|\right]^{-1} \Delta \mathbf{P}_{i}^{\mathbf{g}}} \\
(i=1,2)
\end{array}
$$

Suppose $\Delta \mathrm{X}=\Delta \mathrm{Y}=\Delta \mathrm{Z}$; if we want to achieve tolerant error limits of $\delta, \beta, \gamma,\left[\left|\partial \mathbf{P}_{i}^{\mathbf{g}} / \partial \delta\right|\left|\partial \mathbf{P}_{i}^{\mathbf{g}} / \partial \beta\right|+\left|\partial \mathbf{P}_{i}^{\mathbf{g}} / \partial \gamma\right|\left|\partial \mathbf{P}_{i}^{\mathbf{g}} / \partial \theta_{i}\right|\right]^{-1}$ must be the minimum value.

The attitude of a shearer corresponds to the geological conditions of a mining area, that is, the pitch angle $\beta$ corresponds to the floor gradient along the working face and the roll angle $\gamma$ corresponds to the floor gradient along the faceadvance (retreat) direction. The heading angle corresponds to the angle between open-off cut and north. Suppose the heading angle of a shearer varies within a certain range. The angle between the arms and mainframe $\theta_{i}(i=1,2)$ is determined by the shearer itself. It can be concluded that the range of variables such as $\delta, \beta, \gamma$, and $\theta_{i}$ are available from the geological survey report and the mechanical structure of a shearer, which will be treated as constraint conditions of this GA. 


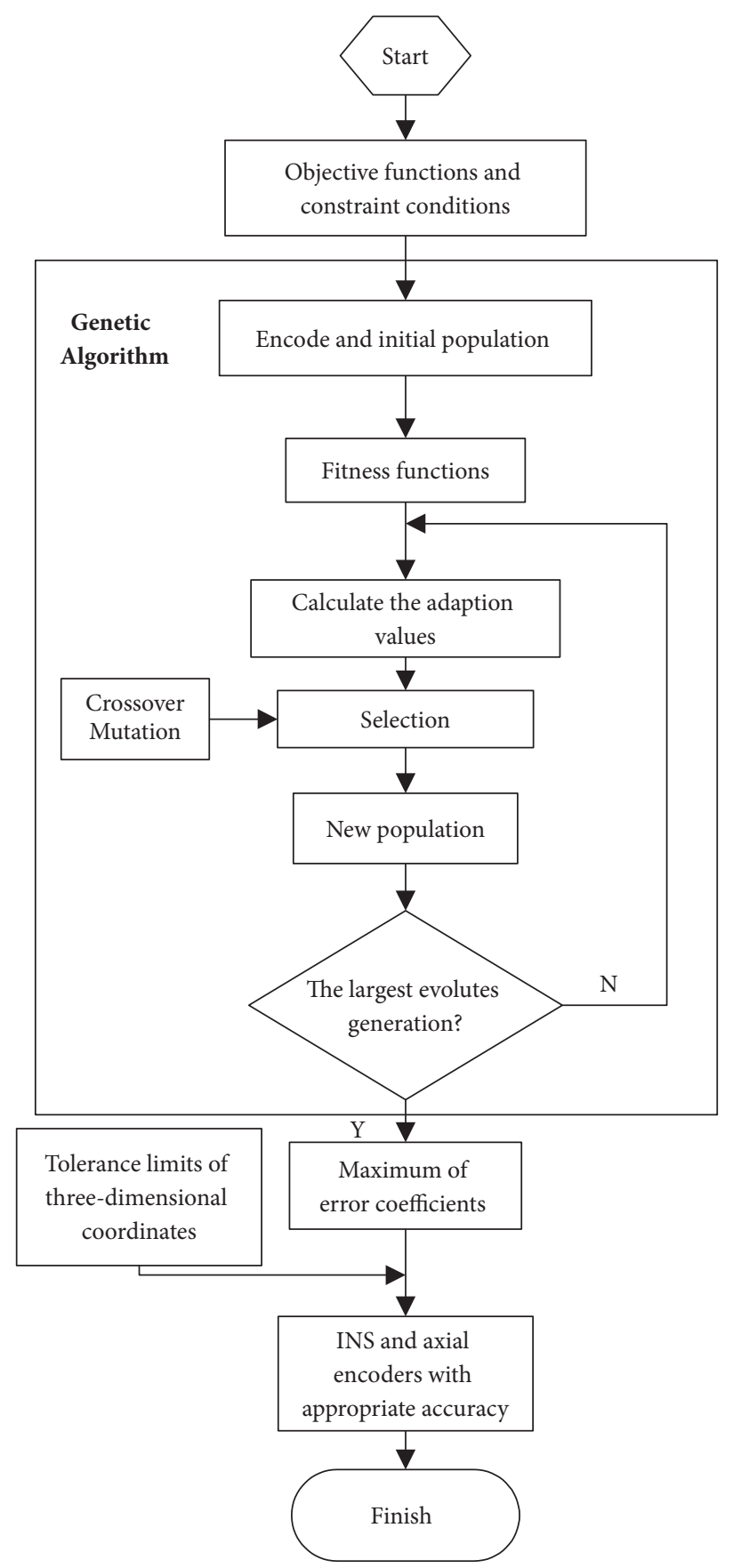

FIGURE 4: The workflow of error analysis with GA [2, 3].

3.2. The Calculation of Error Coefficients Based on GA. Standard optimization process of GA can be divided into five key steps: encoding, selecting, crossover, mutation, and fitness judgment.

Encoding is defined as the mapping relation from problem space to encoding space. The selecting process is choosing individuals with higher fitness value to form a mating pool. Probability of being selected for each chromosome is determined by the proportion of the individual fitness and the total. And the roulette method is mostly applied in the selecting process. For a given group $P=\left\{a_{1}, a_{2}, \ldots a_{n}\right\}$, where $n$ is the scale number of the group, the fitness value of $a_{j}$ can be described as $f\left(a_{j}\right)$ and the probability $p_{s}\left(a_{j}\right)$ can be calculated as follows:

$$
p_{s}\left(a_{j}\right)=\frac{f\left(a_{j}\right)}{\sum_{i=1}^{n} f\left(a_{i}\right)}
$$


where $j=1,2, \ldots n$. The expectation number $P\left(a_{j}\right)$ of previous chromosome in offspring is determined as follows:

$$
P\left(a_{j}\right)=n \cdot p_{s}\left(a_{j}\right)
$$

The crossover in GA is transmitting favorable gene to the next generation by simulating the gene recombinant process of sexual reproduction. Assume that $\left(V^{f}, V^{m}\right)$ were parent chromosomes and the crossover operation was performed on $i$-th position. So the crossover position of the corresponding child chromosomes $\left(V^{s}, V^{d}\right)$ can be presented as follows:

$$
\begin{gathered}
v_{i}^{s}=\alpha v_{i}^{f}+(1-\alpha) v_{i}^{m} \\
v_{i}^{d}=\alpha v_{i}^{m}+(1-\alpha) v_{i}^{f}
\end{gathered}
$$

where $\alpha$ is a random value in the range of $(0,1)$ and $i$ is an integer.

Structure and physical properties of the chromosomes were changed through the mutation operation. Assume that $V^{a}$ was the selected chromosome and the mutation process was performed on $i$-th position, which can be presented as follows:

$$
v_{i}^{a^{\prime}}=\beta v_{i}^{a}+(1-\beta) v_{i}^{b}
$$

where $\beta$ is a random value in the range of $(0,1)$.

As an important evolution algorithm form, GA is used to yield maximum values of error coefficients based on objective functions and constraint conditions as mentioned above. The detailed calculation process with GA is shown in Figure 4 . Firstly, these parameters $\left[\delta, \beta, \gamma, \theta_{i}\right]$ are encoded as double-string, and an initial population is generated at random within the constraint conditions. Secondly, objective functions are treated as fitness functions, and values of these fitness functions are calculated as follows:

$$
\begin{aligned}
& f=\frac{1}{1+g} \\
& g=k_{11}^{2}+k_{12}^{2}+k_{13}^{2}+\cdots+k_{33}^{2}
\end{aligned}
$$

where k11, k12, ., k33 are elements of error matrix in (9) and $\mathrm{f}$ reaches the maximum when $\mathrm{g}$ is the minimum.

Thirdly, with a crossover or mutation operator according to the probability of crossover or mutation $P_{c}$ and $P_{m}$, a new population can be generated. Then, the above calculation process is repeated until the generation of evolutes is the largest. Finally, the output values of fitness functions are the maximum values of the error coefficients, and these results will be used to select the sensors (see Section 3.3).

3.3. Selection of Sensors. An INS and axial encoders can be chosen with maximum values of objective functions by giving the tolerance limits of three-dimensional coordinates, and these sensors with reasonable accuracy can guarantee errors of three-dimensional coordinates within tolerance limits in any mining area.

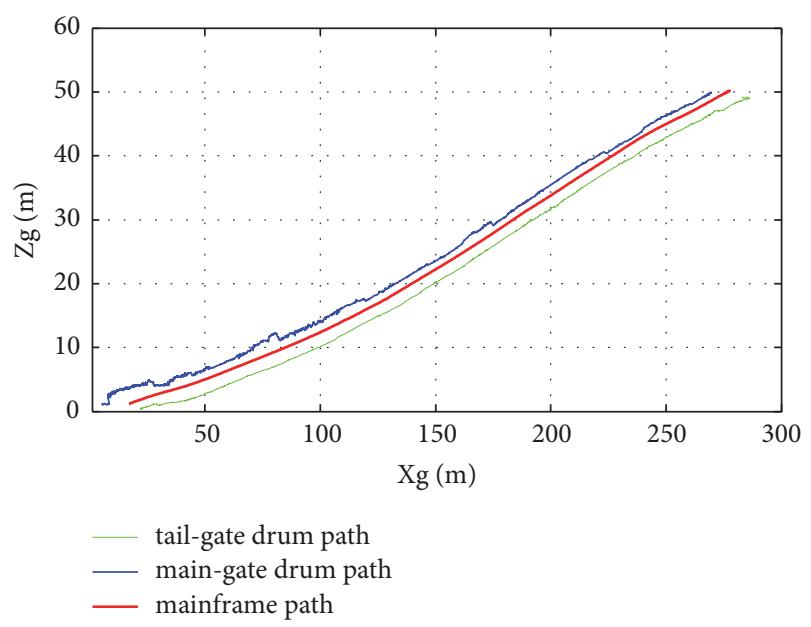

FIGURE 5: Cutting paths of the MG1000/2660-WD shearer with sample data from coal-mining area.

Suppose tolerance limits of three-dimensional coordinates are

$$
\left[\begin{array}{c}
-\Delta X_{i}^{g} \\
-\Delta Y_{i}^{g} \\
-\Delta Z_{i}^{g}
\end{array}\right] \leq \Delta \mathbf{P}_{i}^{\mathbf{g}} \leq\left[\begin{array}{c}
\Delta X_{i}^{g} \\
\Delta Y_{i}^{g} \\
\Delta Z_{i}^{g}
\end{array}\right] \quad(i=1,2) .
$$

Combining with (8) and (10), we get INS and axial encoders with reasonable accuracy:

$$
\mathbf{K}_{i}^{-1}\left[\begin{array}{c}
-\Delta X_{i}^{g} \\
-\Delta Y_{i}^{g} \\
-\Delta Z_{i}^{g}
\end{array}\right] \leq\left[\begin{array}{c}
\Delta \delta \\
\Delta \beta \\
\Delta \theta_{i}
\end{array}\right] \leq \mathbf{K}_{i}^{-1}\left[\begin{array}{c}
\Delta X_{i}^{g} \\
\Delta Y_{i}^{g} \\
\Delta Z_{i}^{g}
\end{array}\right] \quad(i=1,2)
$$

\section{Experimental Results}

Some experiments have been done with a MG1000/2660WD shearer produced by Taiyuan Coal Mining Machinery Company, whose related parameters are $B=1.8 \mathrm{~m}, l=9.82 \mathrm{~m}$, $L=3.54 \mathrm{~m}, 0^{\circ} \leq \theta_{1} \leq 50^{\circ}$, and $-18^{\circ} \leq \theta_{2} \leq 0^{\circ}$. As illustrated in Figure 5 , cutting paths of the shearer were achieved based on the model as follows.

From the geological survey report of the mining area, we can see $\delta \in\left[89^{\circ}, 91^{\circ}\right], \beta \in\left[9^{\circ}, 11^{\circ}\right]$, and $\gamma \in\left[-5^{\circ}, 5^{\circ}\right]$. Combined with the ranges of $\delta, \beta, \gamma, \theta_{1}$, and $\theta_{2}$, maximum values of objective functions can be achieved with GA firstly. The parameters of GA are shown in Table 1. The iteration process of proposed optimization algorithm is presented in Figure 6.

The mechanical parameter of longwall shear was determined by the thickness of coal seam for a shearer cuts the whole thickness of coal seam. So that, three typical longwall shearers working in thin seam, medium-thickness seam, and thick seam separately were selected for verifying the proposed optimization algorithm. Related parameters of the three type 
TABLE 1: Parameters of GA.

\begin{tabular}{lccc}
\hline The group number & The largest evolutes generations & The probability of crossover & The probability of mutation \\
\hline 20 & 100 & 0.5 & 0.01 \\
\hline
\end{tabular}

TABle 2: Parameters of three kinds of shearers.

\begin{tabular}{lccccc}
\hline Model & $B(\mathrm{~m})$ & $L(\mathrm{~m})$ & $l(\mathrm{~m})$ & Angle of ranging arms $\left(^{\circ}\right)$ & Type of seam \\
\hline MG80/200-BW & 1 & 1.41 & 3.8 & {$[-13.2,32.7]$} & Thin $(0.76-1.4 \mathrm{~m})$ \\
MG132/320-W & 1.2 & 1.7 & 5.7 & {$[-15.9,19.6]$} & Medium-thick $(1.3-3.0 \mathrm{~m})$ \\
MG1000/2660-WD & 1.8 & 3.54 & 9.82 & {$[-18,50]$} & Thick $(4.1-6.2 \mathrm{~m})$ \\
\hline
\end{tabular}

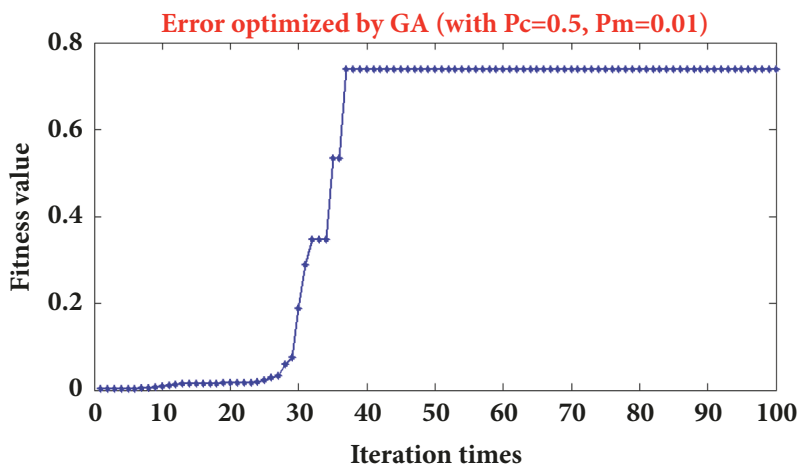

FIGURE 6: Iteration process of GA.

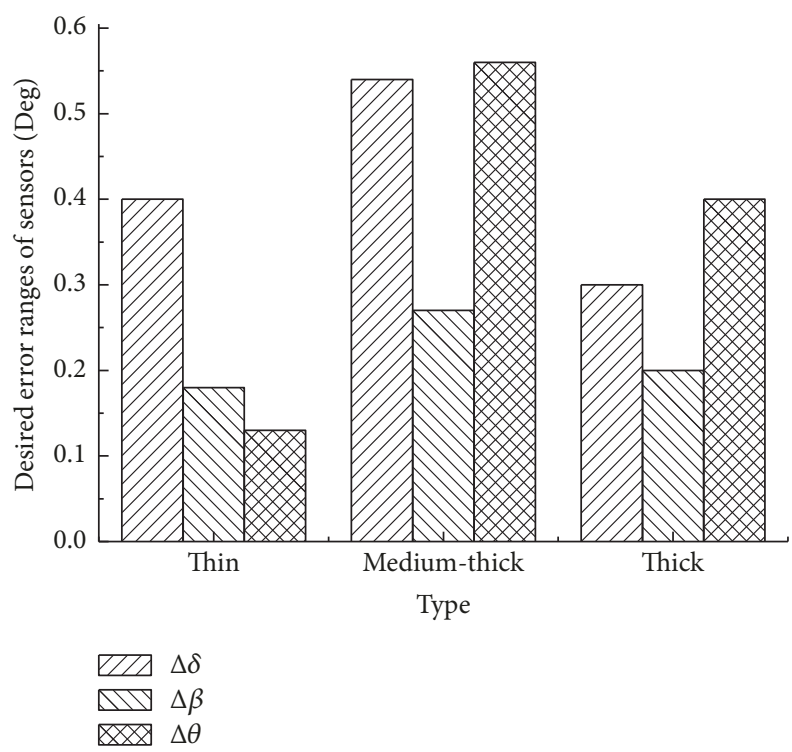

FIGURE 7: Desired error ranges of sensors for three kinds of shearers.

of shearers are shown in Table 2. Usually, tolerance limits of three-dimensional coordinates are within

$$
\Delta \mathbf{P}_{i}^{\mathbf{g}}=\left[\begin{array}{lll}
0.01 & 0.01 & 0.01
\end{array}\right]^{\mathrm{T}} \quad(i=1,2) .
$$

Thus, desired error ranges of sensors mounted on shearers worked in thin seam, medium-thickness seam, and thick seam separately can be achieved with (18). Desired error ranges of sensors for three kinds of shearers are shown in Figure 7. It was concluded that the desired accuracy of the angle between the arms and the shearer mainframe, $\theta$, is higher than the other two angles for a shearer working in thin seam. The geometric dimensioning of a shearer working in thin seam was relatively small. Accordingly, the changing in position of drums caused by the shear's attitude was smaller than that caused by the angle between the arms and the shearer mainframe. This is inverse for the case of medium-thickness seam and thick seam. The larger geometric dimensioning of a shearer working in mediumthickness seam and thick seam induced that the changing in position of drums was more sensitive to the shear's attitude. So the desired accuracy of the pitch angle of a shearer mainframe, $\beta$, is higher than the angle between the arms and the shearer mainframe, $\theta$. According to the measurement principle (Figure 2), an axial encoder and INS can be selected with the desired accuracy exhibited in Figure 7.

\section{Conclusions}

(1) Based on the attitude and the angle between the arms and mainframe, a mathematical model of cutting path of a shearer was built relative to the local geographic coordinate frame.

(2) Considering variables in this mathematical model, errors of main-gate and tail-gate drum positions of a shearer were analyzed based on GA so that suitable and accurate sensors can be chosen according to the desired errors of cutting path.

(3) For the same desired errors of cutting path, the desired accuracy of the angle between the arms and the shearer mainframe, $\theta$, is higher than the other two angles for a shearer in thin seam. For the case of medium-thickness seam and thick seam, the desired accuracy of the pitch angle of a shearer mainframe, $\beta$, is higher.

\section{Data Availability}

The data used to support the findings of this study are available from the corresponding author upon request.

\section{Conflicts of Interest}

The authors declare that there are no conflicts of interest regarding the publication of this paper. 


\section{Acknowledgments}

This research was financially supported by the National Natural Science Foundation of China (U1510116), the National key R\&D Program of China (2018YFC0604503), and the Project Funded by the Priority Academic Program Development of Jiangsu Higher Education Institutions (PAPD).

\section{References}

[1] M. Kelly, D. Hainsworth, D. Reid, C. Caris, and H. Gurgenci, "Longwall automation-an ACARP landmark project," AusIMM Bulletin, vol. 2, pp. 34-37, 2005.

[2] R. L. Haupt and S. E. Haupt, Practical Genetic Algorithms, John Wiley \& Sons, New Jersey, NJ, USA, 2004.

[3] K. Deb, Multi-Objective Optimization Using Evolutionary Algorithms, New York, NY, USA, John Wiley \& Sons, 2001.

[4] S. K. Michael and D. W. Hainsworth, "Outcomes of the landmark long-wall automation project with reference to ground control issues," in Proceedings of the 24th International Conference on Ground Control in Mining, 2005.

[5] J. Ralston, D. Reid, C. Hargrave, and D. Hainsworth, "Sensing for advancing mining automation capability: A review of underground automation technology development," International Journal of Mining Science and Technology, vol. 24, no. 3, pp. 305310, 2014.

[6] J. C. Ralston, "Automated longwall shearer horizon control using thermal infrared-based seam tracking," in Proceedings of the IEEE International Conference on Automation Science and Engineering, vol. 8, pp. 20-25, August 2012.

[7] F. Ren, Z. Yang, and S. Xiong, "Study on the coal-rock interface recognition method based on multi-sensor data fusion technique," Chinese Journal of Mechanical Engineering, vol. 16, no. 3, pp. 321-324, 2003.

[8] B. Wang, Z. Wang, and W. Zhang, "Coal-rock interface recognition method based on EMD and neural network," Journal of Vibration, Measurement and Diagnosis, vol. 32, no. 4, pp. 586590, 2012

[9] J. Sun and B. Su, "Coal-rock interface detection on the basis of image texture features," International Journal of Mining Science and Technology, vol. 23, no. 5, pp. 681-687, 2013.

[10] A. D. Strange, J. C. Ralston, and V. Chandran, "Near-surface interface detection for coal mining applications using bispectral features and GPR," Subsurface Sensing Technologies and Applications, vol. 6, no. 2, pp. 125-149, 2005.

[11] D. C. Reid, D. W. Hainsworth, J. C. Ralston, R. J. McPhee, and C. O. Hargrave, "Inertial navigation: enabling technology for longwall mining automation," 2000, http://scholar.google.com/0.

[12] W. Zhongbin and X. Zhipeng, "Research on the technology of shearer self-adaptive memory cutting," in Proceedings of the Second International Conference on Intelligent Computation Technology and Automation (ICICTA '09), pp. 920-923, Hunan, China, October 2009.

[13] D. Titterton, J. L. Weston, and J. Weston, Strapdown Inertial Navigation Technology, vol. 17, The Institution of Electrical Engineers, Stevenage, UK, 2nd edition, 2004.

[14] W. Guo, N. Zhu, and S. Zhao, "A method of calculation for the shearer drum cutting path," Informatics in Control, Automation and Robotics, vol. 1, pp. 389-396, 2011.

[15] Z. H. Zhu, J. G. Huang, and F. Y. Wu, "A universal proof of error transferring formula of indirect measurement," Journal of Hunan University, vol. 28, no. 3, pp. 1-3, 2001.
[16] Z. A. Kotulski and W. Szczepinski, Error Analysis with Applications in Engineering, vol. 169, Springer Science \& Business Media, 2009. 


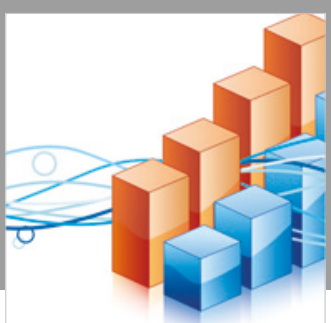

Advances in

Operations Research

\section{-n-m}
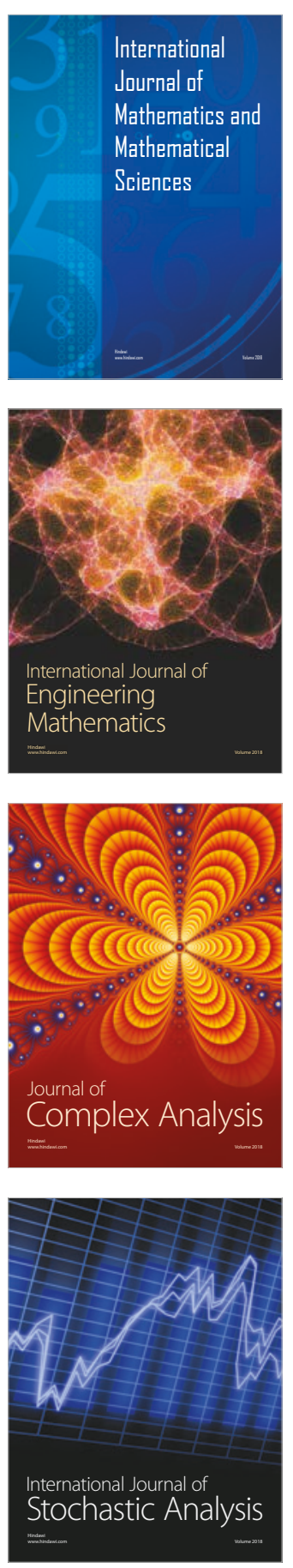
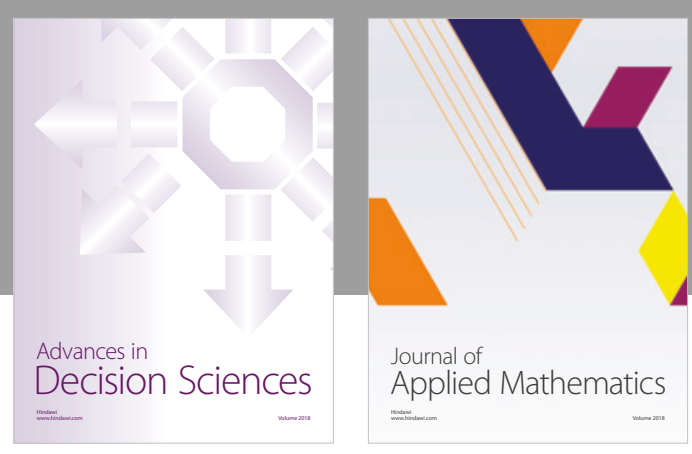

Journal of

Applied Mathematics
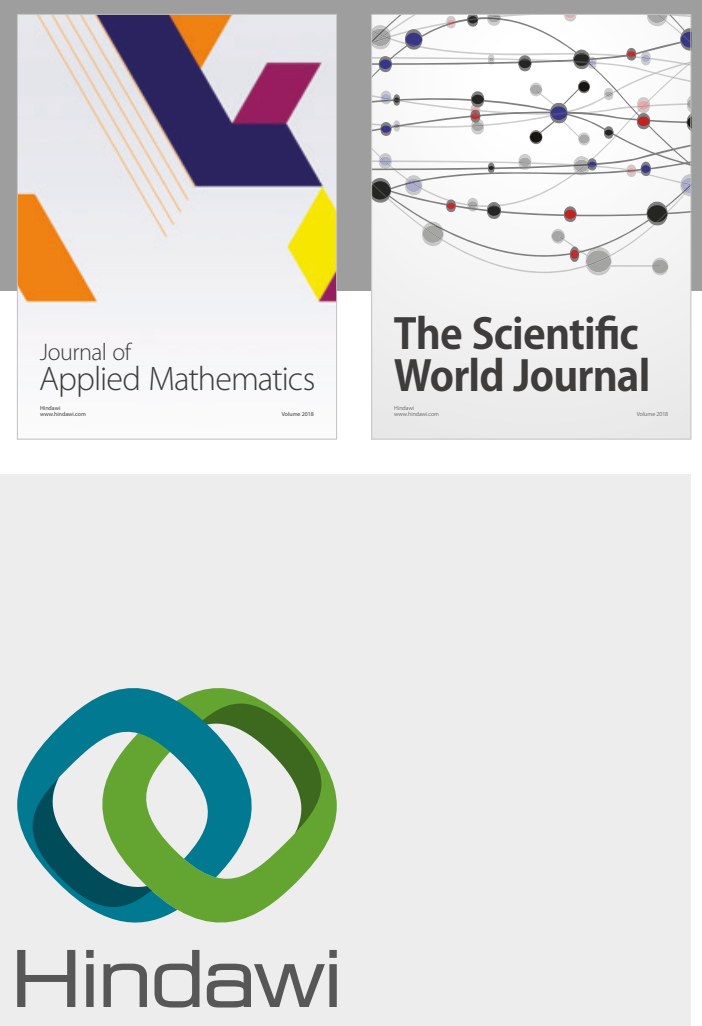

Submit your manuscripts at

www.hindawi.com

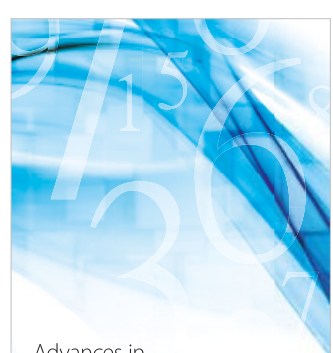

Advances in
Numerical Analysis
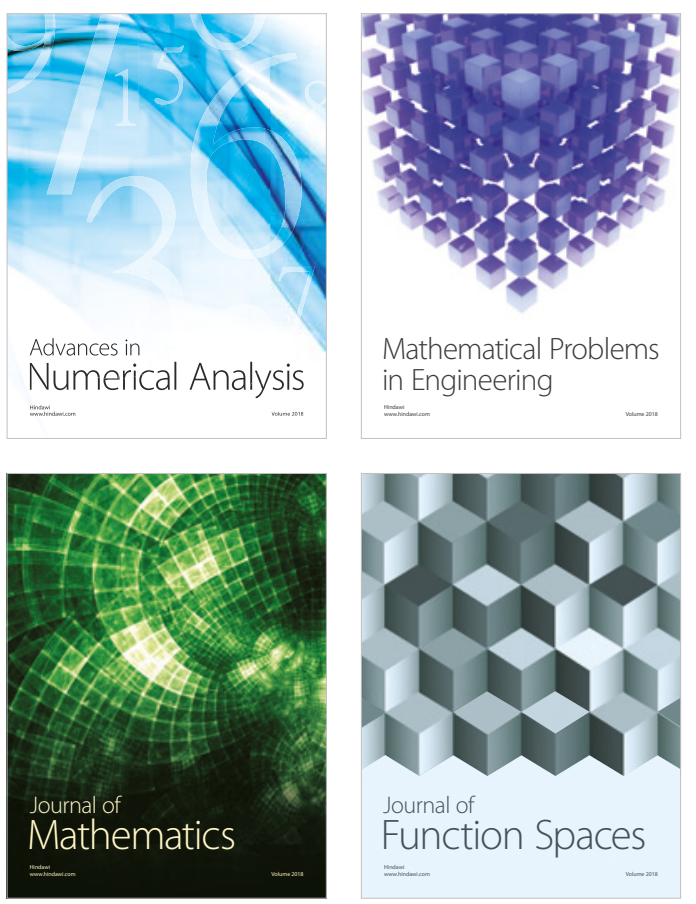

Mathematical Problems in Engineering

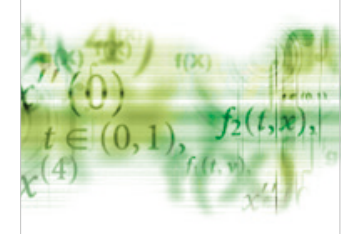

International Journal of

Differential Equations

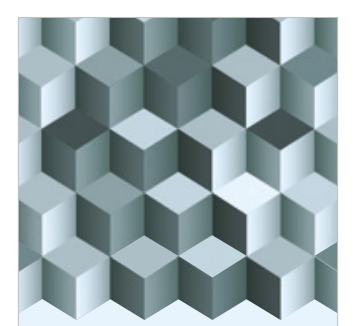

Journal of

Function Spaces

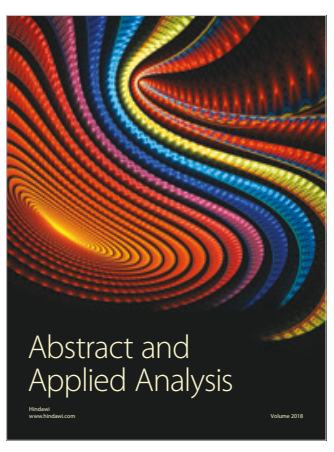

The Scientific

World Journal

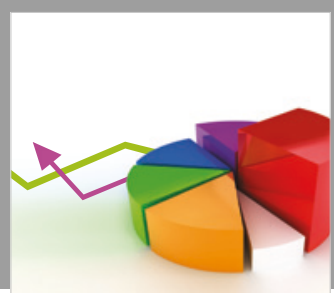

Journal of

Probability and Statistics
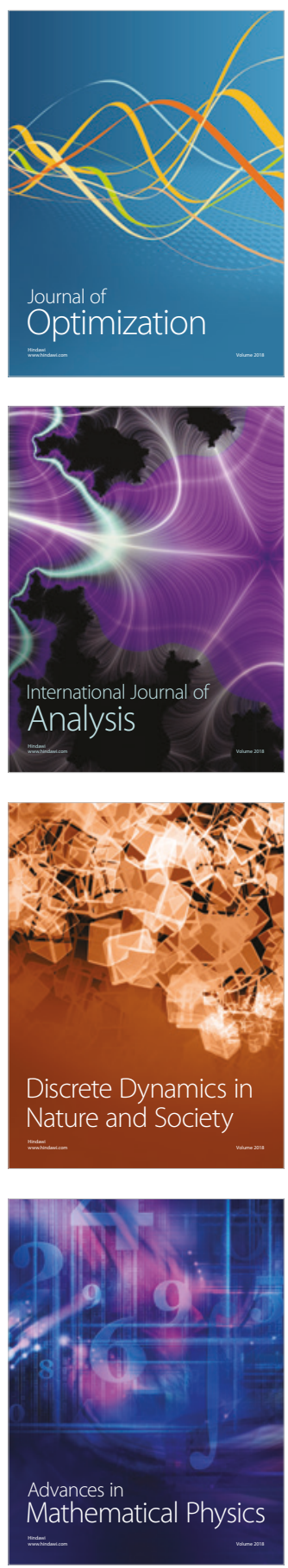